Original Contribution

\title{
DESCRIPTION OF LEARNING CONTENT IN E-LEARNING ENVIRONMENT MOODLE
}

\author{
G. Kiryakova* \\ Faculty of Economics, Trakia University, Stara Zagora, Bulgaria
}

\begin{abstract}
There is an enormous amount of learning materials in Web space, which requires its organization and management in order to facilitate the work of teachers and learners. One key aspect of effective management is to provide means for searching among available learning content based on different criteria. The aim of the current work is to examine and summarize the capabilities of e-learning environment Moodle for description of course items and realize the idea of searching files in repository Server Files by keywords.
\end{abstract}

Key words: learning content, metadata, tags, Moodle, Filepicker

\section{INTRODUCTION}

Educational institutions use learning management systems to implement e-learning. One of the most popular open source system is Moodle. Learning content is structured in courses, which contain learning activities and resources of different types. Over the time, new courses are created and this results in incensement of the available learning content. A consequence of this process is that teachers and learners have difficulties when they search and use content.

Working with many learning materials requires their efficient organization and management. There is a need of searching functionality among the available learning content based on different criteria. Traditionally, this is possible through usage of metadata that means that all content has to be described when it is added to the courses.

\section{PROBLEM}

Unlike commercial learning management systems, Moodle does not offer interaction with metadata. The environment does not provide means for searching and filtering the available content. The reason for this lies in the lack of

*Correspondence to: Gabriela Kiryakova, Faculty of Economics, Trakia University - Stara Zagora,

Bulgaria, gabriela@uni-sz.bg means for describing content, when it is added to a course. The same problem exists with files that are a separate learning resource, and also they can be added to other learning activities. There are no options for files description with appropriate metadata when they are uploaded to the server. The result is growing number of files stored on the server and difficulties with their management.

Moodle 2.x versions have a new strategy in regards of storage and management of files that provide technical enhancements but complicates discoverability of available files stored on the server and reinforces the need to provide search functions within repositories.

\section{CAPABILITIES OF MOODLE FOR DESCRIPTION OF CONTENT (RESOURCES AND ACTIVITIES) BY TAGGING SYSTEM}

Moodle does not provide mechanisms that allow teachers to add metadata both to courses that they create and to resources and activities that are a part of courses. Therefore, it is impossible to track which areas of scientific knowledge available learning resources and activities cover. Recently, the concept of tags is used widely for describing the existing learning content (activities or resources) and facilitating users to navigate and retrieve the necessary content. 
Originally, tag capabilities of Moodle were limited to blog posts (users can add tags that describe the post after its publication in the blog) and user interests (each user can add his interests to his profile and they will be displayed as tags) (1). Now Tagging System includes:

- Course tags. Once courses are created, tags can be added to them. This is a way to describe their content by appropriate metadata. Learners have an access to existing tags and can add new ones related to the course content.

- Quiz question tags. Tags can be added to each quiz question. These metadata improve the search capabilities in the question bank. It is possible to filter and show different categories of questions. The main accent in development process is to create means for searching questions by various criteria, including tags.

\section{RELATED WORKS}

- Tags for resources and activities. Elmadani, Mathews and Mitrovic offer a plug-in that allows adding tags to course content - activities and resources. Teachers can add tags to both resources and activities and determine which areas of knowledge they cover. Adding tags is not possible during the process of creating resources or activities and is performed thereafter. Learners can only view a list of course modules and their tags. Modules are displayed as hyperlinks with associated tags and after clicking they lead to the resource or activity. It is not possible to search or navigate among existing tags (2).

- Shared resource module. The module duplicates the strategy related to resources, known from Moodle 1.x versions, but with opportunities for better treatment of added resources in terms of indexing and cataloging. Indexing of files can be done according to LOM, LOMFR, ScoLOM, SupLOMFR or any other standard based on Dublin Core. There is a search engine based on input metadata (3).

- Course Content Filter. Goslin and Hofmann offer Course Content Filter in Moodle that allows students to search learning objects according to their needs. Searching in the existing materials is based on the use of metadata. When learning objects are added to courses there are options for their categorizing and associating with relevant keywords specified by the administrator of the course. Afterwards these keywords can be used to search learning objects. There are also opportunities for applying filter that shows only those learning objects that are not viewed, respectively downloaded by students (4).

\section{FILES AND REPOSITORIES IN MOODLE}

Files are learning resources that can be included in courses. On the other hand, usually there is an option to add files of different types to learning activities.

The model of working with files in Moodle 1.x differs significantly from that in Moodle 2.x. All files in Moodle before version 2 are uploaded and stored in a physically existing folder known as "Course files" area. There is an area for storing files for each course. This area is used not only for files uploaded by teachers which are a part of the learning content, but also for files uploaded by students which can be assignments or forum attachments. The main limitation of the model is that files are not accessible from other courses and it is necessary to upload the same files for each course (1).

File system in Moodle 2.0 is abstract and files are not stored on the server in a logical tree structure and with the same names as they are on the personal computers. Moodle 2.x centralizes the storage of files in a folder moodledata/filedir and a single database table. Files are stored and saved with hashed names. The actual file names, their metadata and location are stored in a database table 'files' (5).

This new model overcomes the limitations of the previous one (1): eliminate duplication of files - files are stored ones on the server and there are virtual copies for users; re-usability - the same file can be used in various courses, because it is stored independently of the course, security the access to the files is managed in the same way as the access to the activities, they are attached to; integrity - if an activity is imported into another course, files attached to the activity are also imported and thus improves the opportunities for reuse of the activities in different courses.

The main disadvantage of this model is that files are stored in a hierarchy that has a logical form, but from a user perspective tracking and accessing files is more difficult.

The new file approach allows using external repositories as a source of learning materials. There are opportunities to enrich learning environments, expand the possibilities for teachers in organizing their courses and using 
content from different sources. The main aim is to achieve a more effective management of the training process.

Standard repositories in Moodle can be divided into two groups $(1,6)$ :

- Internal repositories - internal repositories are rather repositories for files and provide features similar to those of the repositories. They are available by default in Moodle. Users can access files that are stored on the server via repository Server Files. This provides opportunities for teachers to use files from other courses and activities. Furthermore, they have a personal private space for storing files (repository Private Files).

- External/Optional repositories - Moodle 2.x has the capability to integrate large number of external repositories - Alfresco, Box.net, Dropbox, Flickr, Google Docs (Google Drives), Merlot.org, Picasa web album, Amazon S3, Wikimedia, Youtube videos and more. This is a prerequisite for usage of materials from different sources. External repositories may be enabled by a site administrator.

External repositories provide search functions. Unfortunately, internal repositories, in particular Server Files, do not offer such functions. Searching files on different criteria or keywords is not available. The reason for this is that when files are uploaded there is no option for their description with metadata, which can be used subsequently for searching.

All active repositories are available in File picker. File picker allows easy access to internal and external repositories of files when a user adds a resource or activity with attached files.

When a new file is uploaded to the server, File picker window contains following fields: Save as; Author and Choose license. The latter two are considered as metadata to describe the file. The default value in field Author is the name of the user who uploads a file. The field Select license type is a drop-down list and user can choose the appropriate license. These metadata cannot be used to browse and search through all available files.

The lack of opportunities to search in all available files greatly hampers the work of teachers. Our idea is to overcome this problem by implementing the following suggestions:

1. Adding new fields in File picker window that describe files - when a file is uploaded to the server users fill additional information about it.
2. Implementing search functionality within repository Server Files.

\section{REALIZATION}

1. We added three new fields - Title, Description and Keywords, which can be regarded as descriptive metadata about files. When users upload a file using File picker they can fill data not only about Author and License, but also about Title, Description and Keywords (Figure 1). All fields are not required. The field Title contains information about the title of the file. This title can be different from the file name or they may overlap. From a consumer perspective Title gives a clearer idea about the file content. The field Description contains information which may include educational/pedagogical characteristics of the file. The field Keywords contains keywords describing the files that are specified by users. These three fields provide enough metadata to support basic discoverability of the stored files.

The information in new fields (where it is available, i.e. users filled it in) is displayed when files are selected and viewed and can be changed (Figure 2).

2. The option for searching files in internal repository Server Files is activated. By default, for internal repositories the option for searching files is deactivated.

3. Users can type and search by keywords. The result contains only those files that meet the criteria (Figure 3). We developed a procedure for searching files by keywords within the repository Server Files.

\section{CONCLUSION}

Reusability of the learning resources enhances the efficiency of development of new educational materials. This requires their effective organization, management and retrieval from repositories where they are stored.

The proposed and implemented search functionality within Moodle Server Files repository facilitates teachers during the process of developing new learning content - activities, resources and courses.

The idea can be extended to providing opportunities for multiple criteria search that would limit the number of the displayed files and will facilitate even more teachers. 
If Recentfiles

U Upload a file

\ URL downloader

fo Private files

(3) Wikimedia

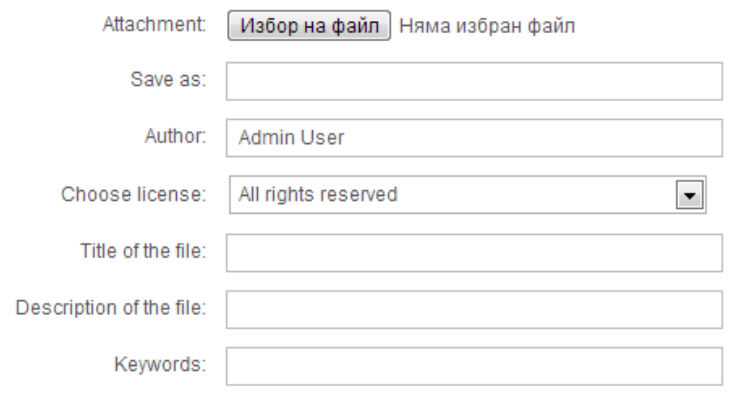

Upload this file

Figure 1. File picker window with added new fields.

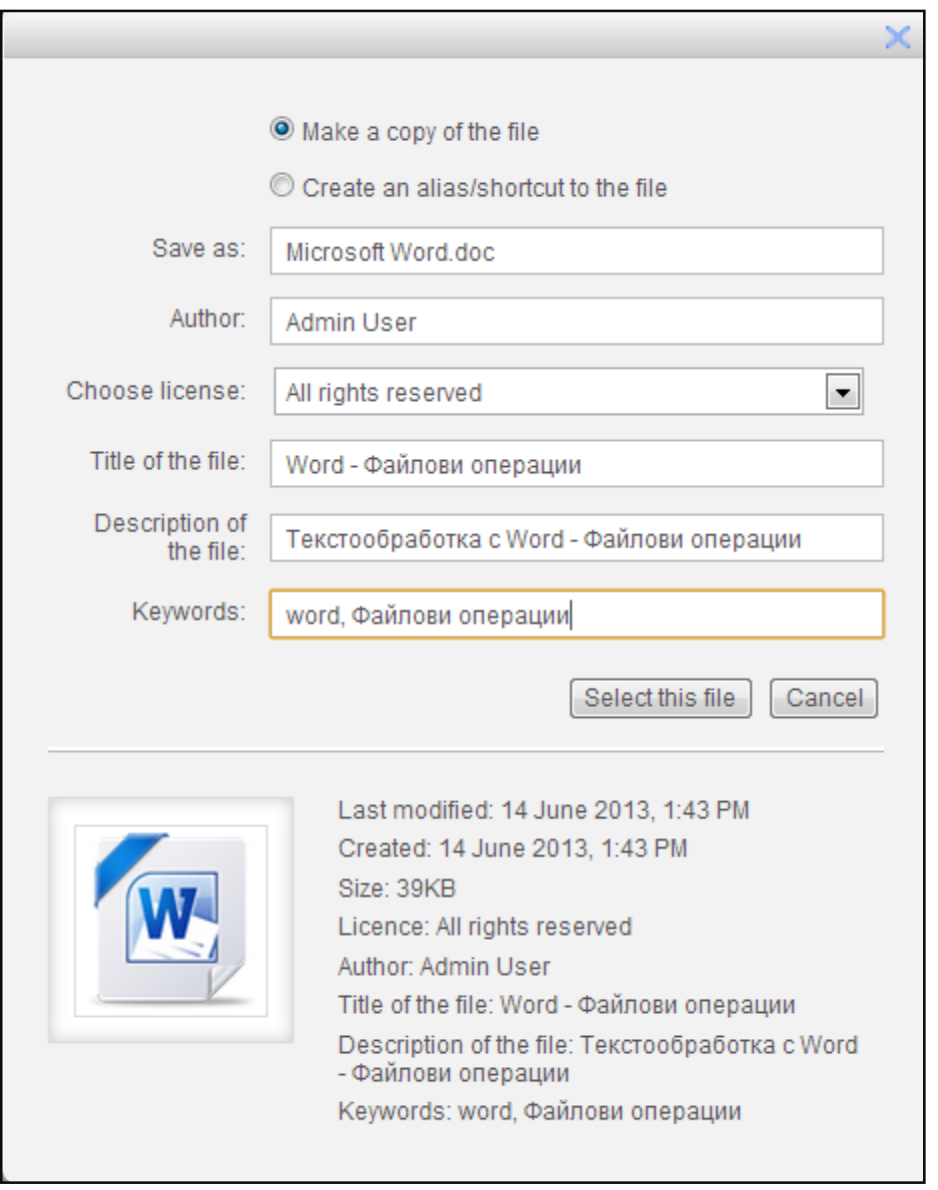

Figure 2. Preview of the information when the file is selected. 


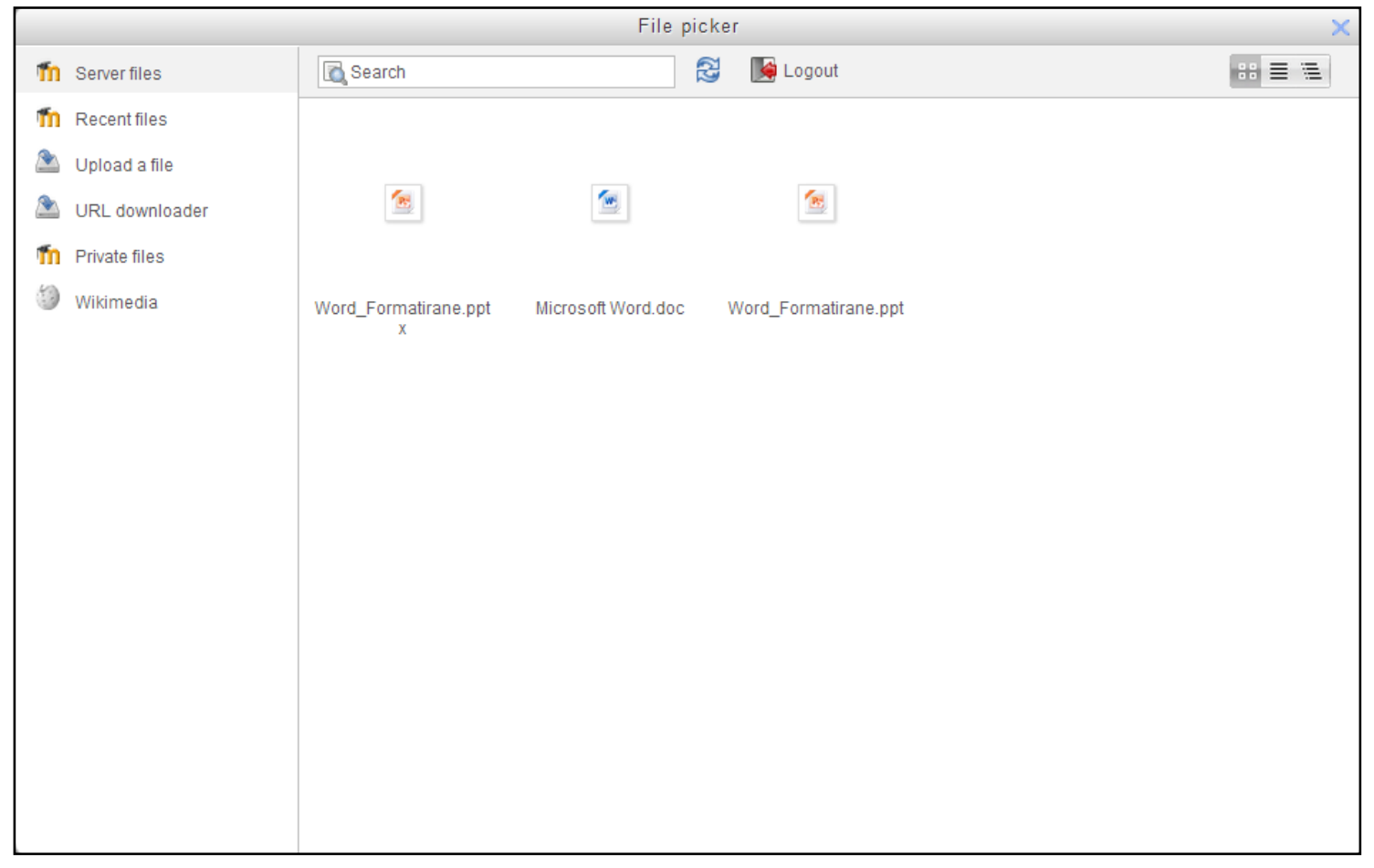

Figure 3. A result of searching files by keyword.

\section{REFERENCES}

1. Moodle site, http://docs.moodle.org/ (last access 02.10.2013)

2. Elmadani, M., Mathews, M. \& Mitrovic, A. (2012), Concept Tagging in Moodle. In Proceedings of the 1st Moodle Research Conference (MRC2012), Retalis, S. \& Dougiamas, M. (Eds), 53-60.

3. MyLearningFactory, http://www.mylearningfactory.com/course/vi ew.php?id=136\&lang =en (last access 02.10.2013)
4. Goslin, K., Hoffmann, M., Development of a Moodle course content using meta data, 9th. IT \& T Conference, 2009

5. Moodle 2.0: File management and repository - file picker vs course files http://www.mattcornock.co.uk/blog/matt/moo dle-2/file-repository-course-files (last access 02.10.2013)

6. Gavin Henrick, Moodle 2 Repositories White Paper, 2011, http://www.academia.edu/1009270/A_look_a t_Moodle_2_Repositories (last access 02.10.2013) 